\section{Intraocular pressure during haemodialysis: a review}

J Levy', D Tovbin², T Lifshitz', M Zlotnik² and $\mathrm{Z}$ Tessler ${ }^{1}$ osmolality, and urea concentration), time of measurements, subgroup of patients, results, and hypothesis of IOP changes during HD can be found in the reported works.

Reported findings, theories, and conclusions are very different, and in some cases, even when a new mechanism for HD influence on IOP was proposed, a critical revision of the previous reports was not performed. To the best of our knowledge, a revision of the issue has not been published yet.

The objectives of this article are to resume the reported evidence of IOP changes during HD, to review the proposed hypothesis of $\mathrm{HD}$ influence on IOP, and to determine if ophthalmic examination is imperative in HD patients.

\section{Methods}

The peer-reviewed literature was analysed and all possible relevant articles were selected. The literature search was conducted in September 2003, and was limited to articles published in English. The terms used for search were intraocular pressure, haemodialysis, dialysis, and intraocular pressure changes. The reference lists from the relevant articles found in the literature search were also included for analysis.

From each article, the following data were included for study: number of patients included in the study; dialysis type; IOP measurement technique; IOP changes during HD; parameters other than IOP investigated in the study: (a) ocular parameters: gonioscopy, visual field, anterior chamber depth, aqueous flow, outflow facility, outflow resistance, corneal and lens thickness, and optic nerve, and (b) HD-related parameters: serum osmolality, serum urea concentration, serum $\mathrm{pH}$, body weight, diastolic pressure, blood bicarbonate, plasma colloid osmotic pressure, plasma $\mathrm{CO}_{2}$ pressure,
${ }^{1}$ Department of

Ophthalmology, Soroka University Medical Center, Beer-Sheva, Israel

${ }^{2}$ Department of Nephrology, Soroka University Medical Center, Beer-Sheva, Israel

Correspondence: J Levy, Department of Ophthalmology, Soroka University Medical Center, PO Box 151, BeerSheva 84101, Israel Tel: + 97286400379 ; Fax: + 97286403927 . E-mail: ljaime@ bgu.ac.il

Received: 13 June 2004 Accepted: 23 September 2004

Published online:

5 November 2004

The authors have no proprietary interest in any of the materials or techniques used in this study 
haematocrit, and postdialysis urea rebound; time of measurements (before, during, and after HD procedure); subgroups of analysed patients; results; statistics performed on results; proposed hypothesis for explanation of results; proposed treatment for IOP rise during HD; agreement or disagreement with previous articles; if a more general theory for HD influence on IOP was suggested; and finally if guidelines were proposed for ophthalmic examination in patients undergoing HD.

\section{Results}

\section{Number of patients}

The number of patients undergoing HD in whom IOP changes were studied varied from 1 to 55 (19. $4 \pm 14.5$, average $\pm S D$ ). The median was 17.5 patients. In seven works, ${ }^{2-8}$ examinations were performed in more than 30 patients. Five articles presented the findings in only one case. During the last 5 years, four articles ${ }^{9-12}$ were published in the ophthalmic and nephrologic literature presenting the findings of acute IOP rise in single HD patients.

\section{Dialysis type and its influence on IOP changes}

Till the early 1980s acetate dialysers were predominantly used, and gradually replaced by bicarbonate dialysers.

The influence of dialyser type on IOP changes was investigated by Rever et $a l^{13}$ who studied IOP and anterior chamber depth (ACD) in 14 patients during both acetate and bicarbonate HD. IOP did not change in any patient during either acetate or bicarbonate in the dialysate. However, ACD decreased significantly during acetate but not bicarbonate HD. The authors suggested that the probable explanation of these findings is similar to that for the dialysis disequilibrium syndrome. That is, as urea is removed from the intracellular compartment, there may be a generation of idiogenic osmols, resulting in a lowering of intraocular $\mathrm{pH}$, which in turn may have a local effect on formation of aqueous humour. Acetate HD may result in a longer period of acidosis. On the other hand, bicarbonate HD, which is associated with a steadily rising blood $\mathrm{pH}$, may more rapidly correct the intraocular acidosis and thus permit normal aqueous dynamics to proceed. The authors concluded that acetate dialysis might adversely affect ocular dynamics in susceptible patients with glaucoma or recent ocular surgery. In such individuals, administration of mannitol or use of bicarbonate dialysate should be considered.

\section{IOP measurement technique}

IOP was measured by Goldmann, 3,6,7,10,12,14 Perkins, 5,15 Schiötz, ${ }^{4,16-18}$ pneumotonometers, ${ }^{19}$ and Tonair ${ }^{20}$ applanation tonometers. In one article, ${ }^{13}$ IOP measurement technique was not detailed in the methods section.

To the best of our knowledge, comparison among different IOP measurement techniques in patients undergoing HD has not yet been performed. Furthermore, the possible effect of IOP measurement technique on different published results has not been discussed earlier either. IOP is a dynamic phenomenon varying with many factors, and its measurement can be affected by the technique of measurement, position of the patient, and blood pressure changes. The Schiötz tonometer ${ }^{21}$ is prone to artefacts caused by abnormal ocular rigidity such as that occuring in myopic eyes; since the refractive state of the studied eyes was not detailed in any study, we ignore if any significant error in IOP measurement occurred. Although currently the most common way to assess IOP is with the slit-lamp mounted Goldmann applanation tonometer, ${ }^{22}$ it is a technique not exempt from errors, such as changes in blood circulation as in Valsalva maneuver, or in overweight patients. The Goldmann applanation technique requires the patient to sit, even when measured with a slit-lamp mounted in a movable platform. Many patients cannot sit up during HD because of hypotension, and if they can do so, systemic blood pressure changes can affect the IOP reading. The Perkins tonometer can be used in recumbent patients, is less influenced by changes in venous pressure than the Goldmann tonometer, and shows a good reliability. ${ }^{23}$ Finally, the Tono-Pen appalantion tonometer, which has been shown to be as accurate as the Goldmann applanation tonometer when measuring IOP in adults with normal values, ${ }^{24}$ has not been evaluated in patients during HD.

\section{IOP changes during HD}

Basically, all the published works can be divided in to three categories: (a) reports showing IOP rise during or after HD; (b) reports showing IOP decrease during HD; and finally (c) articles in which no change in IOP was found:

(a) Reports showing IOP increase during HD: In 1964, Sitprija et $\mathrm{l}^{1,2}$ were the first who studied the changes in IOP during HD. They observed increases in IOP of $41.8 \%$ of control values during HD in uraemic dogs, and an average increase in IOP of $4-8 \mathrm{mmHg}$ in the majority of uraemic patients. ${ }^{1}$ They also found a mean rise in IOP of $5.9 \mathrm{mmHg}$ within $3 \mathrm{~h}$ in patients undergoing HD. ${ }^{2}$ 
After 2 years, Watson and Greenwood ${ }^{25}$ found an average increase of $8.1 \mathrm{mmHg}$ in 11 patients during HD. Burn ${ }^{16}$ observed an IOP rise in one-third of the patients treated with long-term HD.

Gafter $e t a l^{3}$ found IOP increase following HD in only three of the 30 patients, one of them with known glaucoma. In the other 27 patients, IOP slightly increased from $11 \mathrm{mmHg}$ before HD to $12.5 \mathrm{mmHg}$ after HD, both values being lower than the average IOP found in nonuraemic patients.

Cecchin et $\mathrm{al}^{4}$ found IOP increase in $18 \%$ of patients during HD. Gonioscopy revealed narrow angles in all these patients.

Leiba et $a l^{7}$ showed a slight but nonsignificant rise of IOP of $0.35 \mathrm{mmHg}$ when measured after HD, and a significant drop of $-1.48 \mathrm{mmHg}$ for ultrafiltration.

Tawara et $a l^{19}$ found a significant increase of IOP in five eyes with compromised aqueous outflow facility, whereas in eight eyes with normal aqueous outflow facility, IOP did not change significantly.

Tovbin et $a l^{15}$ found that IOP increase in seven of 19 patients with relatively higher postdialysis urea rebound and relatively lower intradialytic change in haematocrit.

(b) Articles reporting IOP decrease during HD: Gutmann and Vaziri ${ }^{18}$ compared IOP values obtained in dialysis patients and compared them with a normal control group. IOP values were significantly lower in HD patients. An insignificant decrease in IOP was noted during the first $2 \mathrm{~h}$ of dialysis. This was followed by a slight rise above the baseline by the end of dialysis.

Costagliola et al ${ }^{5}$ observed after HD a significant decrease in IOP $(19.2 \pm 2.1$ to $14.6 \pm 2.2 \mathrm{mmHg})$.

Tokuyama $\mathrm{et}^{\mathrm{f}} \mathrm{l}^{8}$ found that IOP decreased significantly (change in $-1.8 \mathrm{mmHg}$ ) after HD.

(c) Reports in which IOP did not change during HD: In the early 1970s, Ramsell et al ${ }^{20}$ measured IOP prior to starting IOP and hourly thereafter. They found that IOP did not increase continuously despite a steady decrease in osmolality; rather, IOP fell during the first $2 \mathrm{~h}$, rose during the second and third hours, and then remained constant from the third hour onward.

In 1981, Rever et $a l^{13}$ reported that IOP did not change following a 4-h HD session with either acetate or bicarbonate in the dialysate.

De Marchi et $a l^{6}$ observed that in the majority (41 of 55 patients) they studied, IOP remain unchanged. In 10 patients, there was an excessive rise in IOP (7.8$12.5 \mathrm{mmHg}$ ), and in four patients IOP decreased (3.1$5.1 \mathrm{mmHg}$ ).

In 1990, Austin et $a l^{26}$ reported that IOP did not change significantly in any of the 16 patients during or following HD, despite the marked change in osmolality.

Hojs and Pahor $^{27}$ did not find statistically significant differences in IOP before and after HD.
To date, the last study of IOP changes during HD by Pelit $e a^{28}$ did not show statistically significant differences between IOP before and after HD.

\section{Relationship between other ocular parameters than IOP and IOP changes during HD}

Besides IOP, only in a few articles were other ocular parameters examined, and a possible relationship with IOP changes suggested.

(a). Anterior chamber depth (ACD): Rever et al ${ }^{13}$ reported that ACD decreased significantly during acetate but not bicarbonate HD, and could be maintained during acetate HD by concomitant administration of mannitol. The comparison between ACD and IOP changes was not performed. Costagliola et $a l^{5}$ did not find correlation between IOP and ACD.

(b) Gonioscopy: Jaeger et al ${ }^{17}$ described a patient with narrow angles by gonioscopy who experienced acute rise of IOP during HD. Cecchin et $a l^{4}$ found IOP increase in six patients with narrow angles. De Marchi et $a l^{6}$ observed that in 10 patients with narrow angles, there was an excessive rise in IOP $(7.8-12.5 \mathrm{mmHg})$, whereas in patients with normal angles, IOP remained unchanged or decreased.

(c) Aqueous outflow facility: Only in two works, outflow facility and its relationship with IOP changes was studied. In one of them, ${ }^{29}$ no statistically significant difference was found between outflow facility and IOP changes after HD. In the second work, ${ }^{19}$ in five eyes with severely compromised aqueous outflow facility, the mean per cent change of IOP increased significantly after $90 \mathrm{~min}$, with the exception of the change at $180 \mathrm{~min}$. In eight eyes with normal aqueous outflow facility, the mean per cent change in IOP showed no significant difference at any time during HD.

(d) Visual field (VF): Costagliola et al ${ }^{30}$ studied VF changes over a 5-year period using an Octopus perimeter. They did not find significant differences in the mean sensitivity values of any patient. There was a physiologic decrease of the mean sensitivity, but the mean delta value of the last examination was not statistically different from the first. The authors concluded that HD-induced variations of IOP do not lead to glaucomatous damage in patients with chronic renal failure.

Recently, Pelit et al ${ }^{28}$ compared VF before and after HD using a Humphrey perimeter. Results were not compared over time. Mean deviation (MD) was significantly improved after dialysis, but only slight improvements in pattern standard deviation, corrected pattern standard deviation, and short-term fluctuation were observed. When the authors compared first global indices of the control group with the pre-HD global indices, they noted 
significant difference in MD. The authors recommended VF testing to be performed after HD in patients who are on regular HD program.

(e) Other ocular parameters: Costagliola and Mastropasqua ${ }^{29}$ found that differences in measurements of outflow resistance and corneal and lens thickness before and after HD were not statistically significant.

The status of the optic nerve or retinal nerve fibre layer was not addressed in any of the published reports.

\section{Relationship between HD-induced serum changes and IOP}

(a) Serum osmolality: A discrepancy between the different reported results can be appreciated, with works demonstrating a relationship between serum osmolality and IOP changes, whereas others show the opposite.

Sitprija et $a l^{1}$ observed increases in IOP during HD in uraemic dogs when plasma osmolality decreased by $11 \mathrm{mosm} / \mathrm{kg} \mathrm{H}_{2} \mathrm{O} / \mathrm{h}$, whereas reduction by $8.5 \mathrm{mosm} / \mathrm{Kg}$ $\mathrm{H}_{2} \mathrm{O} / \mathrm{h}$ did not affect IOP significantly. They also described a simultaneous mean decrease of 37.6 mosm in serum osmolality, suggesting a possible relationship between the increase in IOP and the rapid decrease in serum osmolality. ${ }^{2}$

Ramsell et $a l^{20}$ found that serum osmolality fell gradually throughout the HD treatment from $313 \pm 2.5$ to $282 \pm 2.0$ mosm. The changes in IOP and osmolality could not be correlated with one another. Gafter et $a l^{3}$ found no correlation between the changes in IOP and osmolality following HD. IOP did not increase significantly when the change in plasma osmolality during HD was $6 \mathrm{mosm} / \mathrm{l} / \mathrm{h}$. Austin $e \mathrm{al}^{26}$ described a marked change in osmolality after HD $(7.7 \pm 2.2 \mathrm{mosm} / \mathrm{kg} / \mathrm{h})$, which could not be related to IOP changes.

Wizemann and Wizemann ${ }^{31}$ reported no statistical correlation between serum osmolality and IOP.

Leiba et $\mathrm{al}^{7}$ found that serum osmolality decreased significantly when measured after HD, but remained stable for ultrafiltration. The variations in IOP were found to be significantly correlated with changes in osmolality during HD.

In eyes with compromised aqueous outflow facility, Tawara et $a l^{19}$ found that the mean per cent change of serum osmolality decreased significantly after starting dialysis. A negative correlation in the mean per cent change of IOP with serum osmolality was detected. In eyes with normal aqueous outflow, the mean per cent change in IOP showed no significant difference at any time, although the change in serum osmolality decreased significantly.

(b) Plasma colloid osmotic pressure: Tokuyama et al ${ }^{32}$ were the first to study the relationship between plasma colloid osmotic pressure and IOP during HD. They found that during HD, IOP decreased and plasma colloid osmotic pressure increased. They suggested a relationship between plasma colloid osmotic pressure and the change in IOP during HD. In the other work, ${ }^{8}$ they reported a significant increase in plasma colloid osmotic pressure after HD.

No significant correlation was found between the change in IOP and that in plasma osmolarity, whereas the change in IOP was correlated with the change in plasma colloid osmotic pressure and the change in body weight. A significant correlation was found between the change in plasma colloid osmotic pressure and that in body weight.

(c) Postdialysis urea rebound (PDUR) and haematocrit: PDUR, defined as urea measured $1 \mathrm{~h}$ after dialysis subtracted from urea measured at the end of dialysis, was studied by Tovbin et al. ${ }^{15}$ PDUR was positively correlated with mean intradialytic changes in IOP. Intradialytic increase in IOP was observed only in the seven patients with relatively higher PDUR (>9 mg\%), who also had a relatively lower $\%$ intradialytic change in haematocrit $(<8 \%)$. Intradialytic changes in IOP were negatively correlated with $\%$ intradialytic changes in haematocrit in the 12 patients with PDUR $>9 \mathrm{mg}$, and positively correlated with PDUR in the 14 patients with per cent intradialytic change in haematocrit $<8 \%$. The authors suggested that PDUR is strongly correlated with intradialytic changes in IOP, and can predict IOP rise in the presence of lower intradialytic haemoconcentration.

(d) Other parameters: Costagliola et $a l^{5}$ found that no correlation was found between IOP and systolic pressure, diastolic pressure, and bicarbonate. A significant and positive correlation was found between IOP and right atrial pressure. A significant negative correlation was found between IOP and $\mathrm{pH}$. A significant and positive correlation was found between IOP and body weight.

\section{Time of measurements}

Measurements of IOP and other parameters were performed at different times in the reported works. In all the studies, the measurements were taken before and after HD (30 min, $1 \mathrm{~h}$, or one and a half hours). In a few of them, 4,7,8,15,18-20,26 measurements were also performed during HD (every $30 \mathrm{~min}$, hourly, every $2 \mathrm{~h}$, or $2 \mathrm{~h}$ after start). Whether this lack of uniformity at the time of measurements play a role in the discrepancy of results was only mentioned by Leiba et $a l^{7}$ but possible implications could not be found in their work. So we cannot extract any recommendations for time of measurements in HD patients and whether different measurement times during HD can result in different results. To the best of our knowledge, such a work has 
not been performed yet, and its theoretical significance has not been discussed either.

\section{Duration of dialysis}

This is an issue not discussed in the reported studies. The differences in the reported results are in part attributable to the duration of dialysis $(4,5$, and $12 \mathrm{~h}$ ) and the time of measurements cannot be evaluated based on the available information.

\section{Subgroups of patients}

In only two works, ${ }^{6,19}$ patients were divided in to different subgroups, therefore resulting in different findings.

According to gonioscopy features, De Marchi et al divided their patients in to those with narrow angles (Group 1), and those with normal angles (Groups 2 and 3). During HD, IOP underwent an excessive rise (7.8$12.5 \mathrm{mmHg}$ ) in 10 patients (Group 1), remained unchanged (variations below $2 \mathrm{mmHg}$ ) in 41 patients (Group 2), and decreased (3.1-5.1 $\mathrm{mmHg}$ ) in four patients (Group 3).

According to aqueous outflow facilities measured by tonography, Tawara et al ${ }^{19}$ divided their studied eyes into those with severely compromised aqueous outflow facility (Group A), and eyes with normal aqueous outflow facility (Group B). In Group A, the mean per cent change of IOP increased significantly after $90 \mathrm{~min}$, with the exception of the change at $180 \mathrm{~min}$. The mean per cent change of serum osmolality decreased significantly after starting dialysis. A negative correlation in the mean per cent change of IOP with serum osmolality was detected. In Group B, the mean per cent change in IOP showed no significant difference at any time, although the change in serum osmolality decreased significantly.

\section{Statistical analysis}

In the works with the larger number of patients, ${ }^{3-8,13,15,18-20,26-30}$ statistical analysis of the results were performed, resulting in a more supported basis for the proposed conclusions and mechanisms of IOP changes during HD. But in spite of this statistical analysis, opposite findings were found, as we mentioned before.

\section{Proposed theories for explanation of IOP changes during $H D$}

In the first chronological works, increases of IOP during HD were attributed to a rapid decrease in plasma osmolality, ${ }^{1,2}$ or a relative increase in the concentration of urea in the aqueous humor, ${ }^{25}$ possibly resulting from a shift of extracellular fluid from the blood compartment to the anterior chamber, similarly as occurs in the 'dialysisdisequilibrium syndrome'.$^{33}$ The clinical manifestations of this syndrome, such as restlessness, headache, nausea, vomiting, blurring of vision, and muscle twitching, have been attributed to cerebral oedema generated by the 'reverse urea effect': during HD the urea concentration and osmolality in the CSF decreases more slowly than in the blood, causing an osmotic shift of water from the blood into the CSF and a concomitant rise in CSF pressure. Burn ${ }^{16}$ was the first to include the influence of the aqueous outflow facility of the eye in this theory. He concluded that the IOP rise during HD is thought to be part of the cerebral oedema that occurs in the disequilibrium syndrome as a consequence of the rapid drop in serum osmolality. Provided that the eye-outflow system is normal, the rise is small. However, in patients with eye-outflow obstruction due to anterior synechiae or a narrow angle between the iris and the cornea, IOP may rise sharply during HD and lead to acute glaucoma. Later, a similar theory was proposed by Rever et $a l^{13}$ They found that IOP did not change following a 4-h HD session with either acetate or bicarbonate in the dialysate. The authors suggested that the probable explanation of these findings is similar to that for the dialysis disequilibrium syndrome. That is, as urea is removed from the intracellular compartment, there may be a generation of idiogenic osmols, resulting in a lowering of intraocular $\mathrm{pH}$ which in turn may have a local effect on the formation of aqueous humour. Acetate HD may result in a longer period of acidosis. On the other hand, bicarbonate HD, which is associated with a steadily rising blood $\mathrm{pH}$, may more rapidly correct the intraocular acidosis and thus permit normal aqueous dynamics to proceed. They concluded that acetate dialysis might adversely affect ocular dynamics in susceptible patients with glaucoma or recent ocular surgery. Similarly, Leiba et $a l^{7}$ explained slight IOP rises during HD by the dialysis-disequilibrium syndrome, and suggested that these small IOP changes, which are in strong contrast to the marked changes observed in the early publications could be attributable to improved dialysis techniques that allow serum osmolality to be kept relatively stable. Tawara et $a l^{20}$ also proposed that a possible explanation for serious IOP elevation during HD is that dialysis causes a rapid decrease in serum osmolality, resulting in an osmotic gradient between the plasma and intraocular fluids, due to the presence of the blood-ocular barrier. The osmotic gradient draws water from the plasma into the eye. In this situation, if there is no abnormal obstruction in the aqueous outflow pathway, an amount of aqueous humour matching the 
increase in intraocular fluid goes through the pathway out of the eye to maintain the normal level of IOP. In eyes with an obstructed aqueous outflow pathway, however, this compensatory mechanism of aqueous humour drainage does not work well, which results in IOP elevation.

Tokuyama et $a l^{8}$ was the first in evaluating the relationship between the IOP and the plasma colloid osmotic pressure. Plasma colloid osmotic pressure increased significantly after HD. The authors found that the change in IOP during HD was correlated with the change in plasma colloid osmotic pressure and the change in body weight. They suggested that plasma colloid osmotic pressure is instrumental in the hydrodynamic changes that occur during HD.

Finally, Tovbin et $a l^{15}$ implied PDUR in changes of IOP during HD. During the HD procedure, urea removal from the cellular compartment lapses behind its removal from the extracellular compartment, thus creating a gap between cellular and serum urea levels. This gap is reversed by postdialysis urea exit from cells to the extracellular fluid, which is reflected by PDUR. Such a gap may induce intradialysis water movement from the extracellular fluid to the intracellular compartments. The authors suggested that PDUR is strongly correlated with intradialytic changes in IOP, and can predict IOP rise in the presence of lower intradialytic haemoconcentration.

\section{Recommendations for preventing IOP rises during HD}

Intravenous mannitol, ${ }^{17}$ use of bicarbonate in the dialysate, ${ }^{13}$ peritoneal dialysis, ${ }^{6}$ application of hypertonic sodium dialysis, ${ }^{19}$ repairing any aqueous outflow obstruction, ${ }^{19}$ changing the dialysis parameters (by creating conductivity and ultrafiltration profiles and adding a colloid solution at the beginning of the procedure), ${ }^{10}$ and slower urea removal ${ }^{15}$ have all been suggested for preventing a serious rise of IOP during HD. Acetazolamide therapy ${ }^{6}$ can prevent IOP rise, but is not capable of normalizing the IOL level after HD. Moreover, acetazolamide should be avoided in HD patients, owing to metabolic acidosis probably by interfering with extrarenal buffering systems.

\section{Possible guidelines for ophthalmic management of $\mathrm{HD}$ patients}

A close collaboration of ophthalmologists and nephrologists is imperative for treatment of possible acute IOP rises and for long follow-up and prevention of possible optic nerve damage in these patients. If IOP increase during HD is a frequent or infrequent complication was a matter of debate. Anyway, in patients with known glaucoma, narrow angles, recent ocular surgery, or altered aqueous outflow facility, prevention of acute IOP rise during HD by any of the measures described in section 12 seems to be a prudent management. Moreover, the possibility of an obstruction of aqueous humour outflow should be kept in mind in patients showing a significant increase of IOP during HD. Also in these patients only and not in normal eyes, a closer follow-up is desirable, because of greater IOP variations during or after HD. Although long-term changes in the visual field have not been demonstrated in normal patients undergoing $\mathrm{HD}^{6}{ }^{6}$ such as changes that have not been studied in glaucomatous patients, and we ignore their possible implications. As we know that glaucomatous optic nerves are more sensitive to large diurnal IOP fluctuations, theoretically glaucomatous eyes of patients undergoing HD for years can suffer from damage. Older patients with advanced optic disc cupping or advanced visual field defects affecting the central field with split fixation could be at increased risk. Future works on this issue will reveal more information.

The medical team treating the HD patient should be alert on possible ocular symptoms of acute IOP rise, such as blurred vision, eye pain, headache, or signs like middilated pupil and corneal oedema, and consult the ophthalmologist in case of suspected IOP increase.

\section{Conclusion}

In summary, although several works have been examined to date, the relationship and influence of HD on IOP, one can find opposite findings even in recent studies.

It seems to be clear that in patients with glaucoma or with predisposed narrow angles, or eyes with impaired aqueous outflow, the possibility of acute IOP rise during HD could be much more frequent than in normal patients. So in these patients, a more strict ophthalmic scheduled examination seems to be feasible. Reducing large IOP fluctuations during HD is also reasonable.

A number of questions have not been answered yet. First of all, studies examining the long-term influence of HD on optic nerve function such as visual field and ocular blood flow in glaucoma patients have not been performed. More than a decade ago, visual field examinations were compared in normal patients undergoing HD over a 5-year period. ${ }^{30}$ The authors concluded that there is no change in VF parameters. But glaucoma is still a disease of unknown aetiology, and vascular aspects also play a role in its pathogenesis. ${ }^{34}$ To date, studies focusing only on glaucomatous patients undergoing HD have not been presented. An estimated 
2.47 million Americans suffer from primary angle glaucoma, ${ }^{35}$ and about 200.000 are on HD. ${ }^{36}$ Furthermore, end-stage renal disease and glaucoma share common risk factors, such as old age and diabetes mellitus. ${ }^{37}$ So theoretically and although epidemiological data are not available, a considerable number of glaucoma patients are on regular HD. Studies examining HD-induced longterm changes in IOP, optic nerve characteristics, and visual field seem to be necessary.

Second, whether the previous and sometimes opposite reported findings are due to different HD techniques or IOP measurement techniques is a question with an uncertain answer. There is no work comparing these different modalities.

Third, old patients after eye surgery such as cataract extraction are also on regular HD. Possible angle alterations as the result of the surgery can also play a role in IOP changes during HD, and predispose them to optic nerve damage. We are unaware of long-term follow-up studies on HD patients after eye surgery. ${ }^{38}$

And finally, whether there is a real damage to the optic nerve as a consequence of long-term HD even in normal patients is still unknown.

Future studies are needed to answer all these questions. Until then, more frequent ophthalmic examinations on glaucomatous patients undergoing HD could be reasonably managed.

\section{References}

1 Sitprija V, Holmes JH, Ellis PP. Changes in intraocular pressure during hemodialysis. Invest Ophthalmol Vis Sci 1964; 3: 273-283.

2 Sitprija V, Holmes JH, Ellis PP. Intraocular pressure changes during artificial kidney therapy. Arch Ophthalmol 1964; 72: 626-631.

3 Gafter U, Pinkas M, Hirsch J, Levi J, Savir J. Intraocular pressure in uremic patients on chronic hemodialysis. Nephron 1985; 40: 74-75.

4 Cecchin E, De Marchi S, Tesio F. Intraocular pressure and hemodialysis. Nephron 1986; 43: 73-74.

5 Costagliola C, Cotticelli L, Di Benedetto A, Iuliano G, Menzione M, Piccione V et al. The influence of hemodialysis on intraocular pressure: a study of the correlation of selected parameters. Glaucoma 1989; 11: 142-145.

6 De Marchi S, Cechin E, Tesio F. Intraocular pressure changes during hemodialysis: prevention of excessive dialytic rise and development of severe metabolic acidosis following acetazolamide therapy. Renal Failure 1989; 11: 117-124.

7 Leiba H, Oliver M, Shimshoni M, Bar-Khayim Y. Intraocular pressure fluctuations during regular hemodialysis and ultrafiltration. Acta Ophthalmol 1990; 68: 320-322.

8 Tokuyama T, Ikeda T, Sato K. Effect of plasma colloid osmotic pressure on intraocular pressure during hemodialysis. Br J Ophthalmol 1998; 82: 751-753.
9 Choong YF, Menage MJ. Symptomatic acute raised IOP following hemodialysis in a patient with end stage renal failure. Br J Ophthalmol 1998; 82: 1342.

10 Minguela I, Andonegui J, Aurrekoetxea B, Ruiz de Gauna R. Prevention of intraocular pressure elevations during hemodialysis. Am J Kidney Dis 2000; 36: 197-198.

11 Ikeda N, Saito T, Hayasaka S, Hayasaka Y. Unilateral symptomatic elevation of intraocular pressure and prevention using a hyperosmotic agent during hemodialysis. Jpn J Ophthalmol 2001; 45: 659-661.

12 Masuda H, Shibuza Y, Ohira A. Markedly increased unilateral intraocular pressure during hemodialysis in a patient with ipsilateral exfoliative glaucoma. Am J Ophthalmol 2002; 129: 534-536.

13 Rever B, Fox L, Bar-Khayim Y, Nissenson A. Adverse ocular effects of acetate hemodialysis. Kidney Int 1981; 19: 157.

14 Tokuyama T, Ikeda T, Sato K. Effect of plasma colloid osmotic pressure on intraocular pressure during hemodialysis. Br J Ophthalmol 1998; 82: 751-753.

15 Tovbin D, Belfair N, Shapira S, Rosenthal G, Friger M, Feldman $\mathrm{L}$ et al. High postdialysis urea rebound can predict intradialytic increase in intraocular pressure in dialysis patients with lowered intradialytic hemoconcentration. Nephron 2002; 90: 181-187.

16 Burn RA. Intraocular pressure during hemodialysis. $\mathrm{Br} J$ Ophthalmol 1973; 57: 511-513.

17 Jaeger P, Morisod L, Wauters JP, Faggioni R. Prevention of glaucoma during hemodialysis by mannitol and acetazolamide. N Engl J Med 1980; 18: 702.

18 Gutmann SM, Vaziri ND. Effect of hemodialysis on intraocular pressure. Artif Organs 1984; 8: 62-65.

19 Tawara A, Kobata H, Fujisawa K, Abe T, Ohnishi Y. Mechanism of intraocular pressure elevation during hemodialysis. Curr Eye Res 1998; 17: 339-347.

20 Ramsell JR, Ellis PP, Paterson CA. Intraocular pressure changes during hemodialysis. Am J Ophthalmol 1971; 72: 926-930.

21 Brubaker RF, Tonometry In: Tasman W, Jaeger EA (eds) Duane's Clinical Ophthalmology on CD-Rom, Chapter 47, Lippincot Williams \& Wilkins: Philadelphia, 2003 edition.

22 Gonzaga dos Santos M, Makk S, Berghold A, Eckhardt M, Haas A. Intraocular pressure difference in Goldmann applanation tonometry versus Perkins hand-held applanation tonometry in overweight patients. Ophthalmology 1998; 105: 2260-2263.

23 Damji KF, Muni RH, Munger RM. Influence of corneal variables on accuracy of intraocular pressure measurement. J Glaucoma 2003; 12: 69-80.

24 Kao SF, Lichter PR, Bergstrom TJ, Rowe S, Musch DC. Clinical comparison of the Oculab Tono-Pen to the Goldmann applanation tonometer. Ophthalmology 1987; 94: 1541-1544.

25 Watson SG, Greenwood WR. Studies on the intraocular pressure during hemodialysis. Can J Ophthalmol 1966; 1: 301-307.

26 Austin JN, Klein M, Mishell J, Contiguglia SR, Levy J, Chan $\mathrm{L}$ et al. Intraocular pressure during high-flux hemodialysis. Renal Failure 1990; 12: 109-112.

27 Hojs R, Pahor D. Intraocular pressure in chronic renal failure patients treated with maintenance hemodialysis. Ophthalmologica 1997; 211: 325-326. 
28 Pelit A, Zumrutdal A, Akova Y. The effect of hemodialysis on visual field test in patients with chronic renal failure. Curr Eye Res 2003; 26: 303-306.

29 Costagliola C, Mastropasqua L. The influence of hemodialysis on intraocular pressure: III. Aqueous humor dynamics and tissue hydration. Ann Ophthalmol 1991; 23: 31-34.

30 Costagliola C, Di Benedetto A, Piccione V, Rinaldi E. The influence of hemodialysis on intraocular pressure: II. Evaluation of visual field modifications over five years. Glaucoma 1991; 13: 6-8.

31 Wizemann AB, Wizemann V. Effect of serum osmolality, arterial blood pressure and volume loss on intraocular pressure during hemodialysis, hemofiltration and hemofiltration/hemodialysis. Graefes Arch Clin Exp Ophthalmol 1980; 213: 43-47.

32 Tokuyama T, Ikeda T, Ishikawa H, Sato K. Marked decrease in intraocular pressure in a neovascular glaucoma patient during hemodialysis. Jpn J Ophthalmol 1997; 41: 101-103.
33 Wakim KG. The pathophysiology of the dialysisdisequilibrium syndrome. Mayo Clin Proc 1969; 44: 406-429.

34 Chung HS, Harris A, Evans DW, Kagemann L, Garzozi HJ, Martin B. Vascular aspects in the pathophysiology of glaucomatous optic neuropathy. Surv Ophthalmol 1999; 43(Suppl 1): S43-S50.

35 Quigley HA, Vitale S. Models of open-angle glaucoma prevalence and incidence in the United States. Invest Ophthalmol Vis Sci 1997; 38: 83-91.

36 Bommer J. Prevalence and socio-economic aspects of chronic kidney disease. Nephrol Dial Transplant 2002; 17(Supp 11): 8-12.

37 Wallace LM, Award MD. Medical management of glaucoma. N Engl J Med 1998; 339: 1298-1307.

38 Hayashi H, Kurata Y, Imanaga Y, Goya K, Oshima K. Vitrectomy for diabetic retinopathy in patients undergoing hemodialysis for associated end-stage renal failure. Retina 1998; 18: 156-159. 